\title{
Nef Alleles derived from HIV-Positive Patients with Pulmonary Hypertension Affect Production and Function of Exosomes in Endothelial Colony Forming Cells
}

\author{
Arthur Cross-Najafi ${ }^{1}$, Tyler Colbert ${ }^{1,2}$, Sarvesh Chelvanambi ${ }^{1}$, Sharilyn \\ Almodovar $^{3}$, Sonia Flores ${ }^{4}$, Matthias Clauss ${ }^{1,2}$ \\ ${ }^{1}$ Indiana University School of Medicine, ${ }^{2}$ Department of Pulmonary, Critical Care, \\ Sleep \& Occupational Medicine, ${ }^{3}$ TexasTech University, ${ }^{4}$ University of Colorado
}

Background: The HIV positive population has a $>300$ fold higher risk for developing pulmonary hypertension $(\mathrm{PH})$ as compared to the general population. $\mathrm{PH}$ is a disease characterized by elevated blood pressure in the pulmonary vasculature and is currently with no cure. Experiments with primates found that Nef, an HIV encoded protein secreted in exosomes from HIV+ cells, plays an important role in the development of HIV-PH. HIV-Nef+ exosomes induce endothelial dysfunction, including premature senescence of endothelial colony forming cells (ECFCs). We hypothesize that exosome functions and concentrations in HIV+ patients may vary based on Nef-mutations, and thus potentially define a predictor for the risk of developing $\mathrm{PH}$.

Methods: Nef exosomes were isolated from HEK293? cells transfected with molecular constructs containing HIV Nef signature sequences recovered from patients with HIV-PH. The Nef exosomes were then used to treat ECFC. We measured cell proliferation (by MTT assay), apoptosis (cleaved caspase- 3 by Western blot), and cell senescence ( $\mathrm{p} 16^{\text {ink } 4 \mathrm{~A}}$ by flow cytometry) in ECFCs treated with Nef exosomes. LM-10-Nanosight was used to quantify exosome concentrations in patient plasma.

Results: ECFC treated with Nef exosomes induced a 30\% reduction in cell proliferation $(\mathrm{p}<0.05, \mathrm{MTT})$. Western blot analysis demonstrated no significant change in apoptotic signaling, and flow cytometric analysis of $\mathrm{p} 16^{\text {ink4A }}$ showed increased senescence in Nef exosome-treated ECFCs versus mock control. "Nef $\mathrm{PH}$-transfected cells showed that decreased proliferation was dependent on specific mutations of Nef.

Conclusion: Expression of Nef in cells results in increased exosome production and treatment of ECFC with these exosomes resulted in decreased proliferation, which inducting the cells towards senescence based on signature sequences in Nef. 